\title{
Identification of glucocorticoid receptor in Drosophila melanogaster
}

\author{
Gloria Bartolo', Leandra O. Gonzalez', Saleem Alameh', C. Alexander Valencia ${ }^{2,3,4}$ and \\ Mikhail Martchenko Shilman ${ }^{1 *}$ (D)
}

\begin{abstract}
Background: Vertebrate glucocorticoid receptor (GR) is an evolutionary-conserved cortisol-regulated nuclear receptor that controls key metabolic and developmental pathways. Upon binding to cortisol, GR acts as an immunosuppressive transcription factor. Drosophila melanogaster, a model organism to study innate immunity, can also be immunosuppressed by glucocorticoids. However, while the genome of fruit fly harbors 18 nuclear receptor genes, the functional homolog of vertebrate GR has not been identified.

Results: In this study, we demonstrated that while D. melanogaster is susceptible to Saccharomyces cerevisiae oral infection, the oral exposure to cortisol analogs, cortisone acetate or estrogen, increases fly sensitivity to yeast challenge. To understand the mechanism of this steroid-induced immunosuppression, we identified the closest genetic GR homolog as D. melanogaster Estrogen Related Receptor (ERR) gene. We discovered that Drosophila ERR is necessary for cortisone acetate- and estrogen-mediated increase in sensitivity to fungal infection: while ERR mutant flies are as sensitive to the fungal challenge as the wildtype flies, the yeast-sensitivity of ERR mutants is not increased by these steroids. Interestingly, the fungal cortisone analog, ergosterol, did not increase the susceptibility of Drosophila to yeast infection. The immunosuppressive effect of steroids on the sensitivity of flies to fungi is evolutionary conserved in insects, as we show that estrogen significantly increases the yeast-sensitivity of Culex quinquefasciatus mosquitoes, whose genome contains a close ortholog of the fly ERR gene.
\end{abstract}

Conclusions: This study identifies a D. melanogaster gene that structurally resembles vertebrate GR and is functionally necessary for the steroid-mediated immunosuppression to fungal infections.

Keywords: Glucocorticoid receptor, Drosophila melanogaster, Fruit fly, Estrogen receptor, Cortisol, Cortisone, Culex quinquefasciatus, Mosquitoes, Saccharomyces cerevisiae, Infection

\section{Background}

Glucocorticoids (GCs), steroid hormones produced in the adrenal cortex of the kidney [1], are important for regulating numerous physiological functions such as glucose metabolism and immune response [2]. Naturally occurring GCs in the human body are inactive precursor cortisone and its active metabolite, cortisol [1, 3, 4]. Cortisol, converted from cortisone via type $111 ß$-hydroxysteroid

\footnotetext{
*Correspondence: mikhail_shilman@kgi.edu

${ }^{1}$ Henry E. Riggs School of Applied Life Sciences, Keck Graduate Institute, Claremont, CA 91711, USA

Full list of author information is available at the end of the article
}

enzyme, functions by binding directly to the ligand binding domain (LBD) of glucocorticoid receptors (GRs) found within the cytosol of the target cell $[1,3,4]$. Once bound to cortisol, GR translocates from the cytosol to the nucleus where it homodimerizes [3, 4]. The cortisol-bound GR homodimer can act as a transcriptional activator of genes encoding anti-inflammatory proteins by allowing its DNA binding domain (DBD) to bind to glucocorticoidresponsive elements (GREs) [3, 4]. Concurrently, the cortisol-GR homodimer can bind to and inhibit the function of transcription factor Nuclear Factor $\kappa B(\mathrm{NF}-\mathrm{kB})$,

(c) The Author(s). 2020 Open Access This article is licensed under a Creative Commons Attribution 4.0 International License, which permits use, sharing, adaptation, distribution and reproduction in any medium or format, as long as you give appropriate credit to the original author(s) and the source, provide a link to the Creative Commons licence, and indicate if changes were made. The images or other third party material in this article are included in the article's Creative Commons licence, unless indicated otherwise in a credit line to the material. If material is not included in the article's Creative Commons licence and your intended use is not permitted by statutory regulation or exceeds the permitted use, you will need to obtain permission directly from the copyright holder. To view a copy of this licence, visit http://creativecommons.org/licenses/by/4.0/ The Creative Commons Public Domain Dedication waiver (http://creativecommons.org/publicdomain/zero/1.0/) applies to the data made available in this article, unless otherwise stated in a credit line to the data. 
ultimately repressing the synthesis of NF-kB-dependent inflammatory proteins $[3,4]$ (Fig. 1a, left panel).

$\mathrm{NF}-\mathrm{KB}$ is evolutionary-conserved and an integral part of the fruit fly Drosophila melanogaster innate immune response when challenged with entomopathogenic microbes. Upon the detection of microbial pathogens, Drosophila systemic humoral response activates two NF$\mathrm{KB}$-activating pathways, Imd and Toll, leading to the production of anti-microbial peptides (AMPs) $[5,6]$. The Imd pathway is primarily induced by gram-negative bacteria by recognizing DAP-type peptidoglycan via PGRPLC receptors located on the cell surface of enterocytes or fat body cells $[5,7]$. Once the presence of bacteria is detected, the NF- $\mathrm{kB}$ transcription factor Relish activates the transcription of AMPs such as diptericin. A parallel cascade is triggered by the detection of gram-positive bacteria and fungi. The Toll pathway detects lysine-type peptidoglycan of gram-positive bacteria via PGRP-SA and GNBP1, as well as B-glucans on the cell walls of fungi via GNBP3. This triggers the activation of the Toll receptor, and ultimately leads to the nuclear translocation of the NF- $\mathrm{kB}$ transcription factor Dif, activating the transcription of AMPs such as drosomycin [5] (Fig. 1a, right panel). Recent studies show that sensing of the type of the bacterial cell wall is less stringent than previously thought and that both fly pathways are capable of detecting lys- and dap-peptidoglycan based on the accessibility of bacterial cell wall [7].

Human GR is a member of a nuclear receptor (NR) class of proteins. NRs are a superfamily of ligand regulated transcription factors activated by steroid hormones and various other lipid-soluble signals responsible for regulating a variety of processes such as embryonic development and metabolism [8-10]. Additionally, NRs are evolutionary conserved and represented in all animal phyla, including humans and Drosophila [11]. Human NR superfamily includes 48 NRs, which could be divided into six subfamilies based on their sequence similarity. Although Drosophila has only 18 NRs, they represent all 6 sub-families found in humans $[8,12,13]$. A previous study demonstrated that orally administered corticosteroid increases the sensitivity of Drosophila to pathogenic fungus, Rhizopus oryzae [14], alluding to the existence of unidentified fly GR (Fig. 1a). In this study, we report the identification and function of the D. melanogaster GR ortholog by examining yeast infection susceptibility upon steroid treatments.

\section{Results}

Genetic in silico search for GR homolog in the Drosophila melanogaster genome

Mammalian NR members of the same group share at least $80 \%$ identity in DBDs and at least $40 \%$ identity in LBDs [10]. Human GR belongs to the NR subfamily 3 (also known as steroid NRs), which is comprised of a total of nine family members, such as Homo sapiens estrogen-related receptor and estrogen receptor (hsERR1 and hsER1, respectively). Interestingly, Drosophila harbors only one member in the subfamily 3 , which was called D. melanogaster ERR (dmERR) for its sequence homology to hsERR1 $[8,10,15,16]$. We aligned the sequence of dmERR with sequences of several members of human NR subfamily 3 (hsERR1, hsER1, and GR) and observed an overall amino acid homology between 47 to $58 \%$ (Fig. 1b), with high similarity in the DBD (70-96\%) and the LBD (55-57\%) (Additional file 1). Due to a comparable degree of sequence homology of dmERR to GR and to hsERR1 (Fig. 1b), as well as the ability of corticosteroids to immunosuppress flies and humans to fungi [14], we hypothesized that dmERR may function as a functional ortholog of GR.

\section{Susceptibility of Drosophila melanogaster to Saccharomyces cerevisiae oral infection}

Microbial pathogens, both bacterial and fungal, can infect Drosophila via different potential routes. A common route of access to microbial pathogens is the penetration of the gut, as Drosophila is naturally exposed to pathogens when foraging for food sources [17]. In addition to exposure to pathogens, Drosophila can also be exposed to non-pathogenic microbes such as Saccharomyces cerevisiae, a budding yeast that co-habitats with Drosophila in nature $[18,19]$. Since the previous study demonstrated that a medically used GC decreases the survival of Drosophila to a human fungal pathogen [14], we set out to test whether naturally occurring GCs sensitize flies to non-pathogenic yeast, Saccharomyces cerevisiae.

Before the administration of GCs, we first assessed the sensitivity of Drosophila to Saccharomyces via oral exposure. Wildtype Oregon-R Drosophila flies were treated with sucrose solutions containing various concentrations of S. cerevisiae (Fig. 2a and Additional file 2), and compared to the pathogenicity of known entomopathogenic Gram-positive Bacillus cereus and Micrococcus luteus, as well as Gram-negative Escherichia coli and Serratia liquefaciens bacteria [7, 20-22] (Figs. 2b-e, respectively). Surprisingly, despite being known as natural symbionts $[18,19]$, we observed that $S$. cerevisiae is pathogenic to flies of both genders via continuous oral exposure. At $4.17 \times 10^{7}$ yeast cells $/ \mathrm{ml}$ in sucrose solution, fly median survival time (i.e. the time at which $50 \%$ of flies are dead) occurs after $58 \mathrm{~h}$ of exposure in both female and male flies (Fig. 2a). At $1.67 \times 10^{7}$ yeast cells $/ \mathrm{ml}, S$. cerevisiae yields a median survival by $71 \mathrm{~h}$ in female flies (Fig. 2a) and $95 \mathrm{~h}$ in male flies (Additional file 2). A further half-fold decrease in fungal concentration $\left(8.30 \times 10^{6}\right.$ cells $\left./ \mathrm{ml}\right)$ leads to even longer median survival times: $101.5 \mathrm{~h}$ in female flies and $141 \mathrm{~h}$ in male flies (Fig. 2a and Additional file 2, 
a

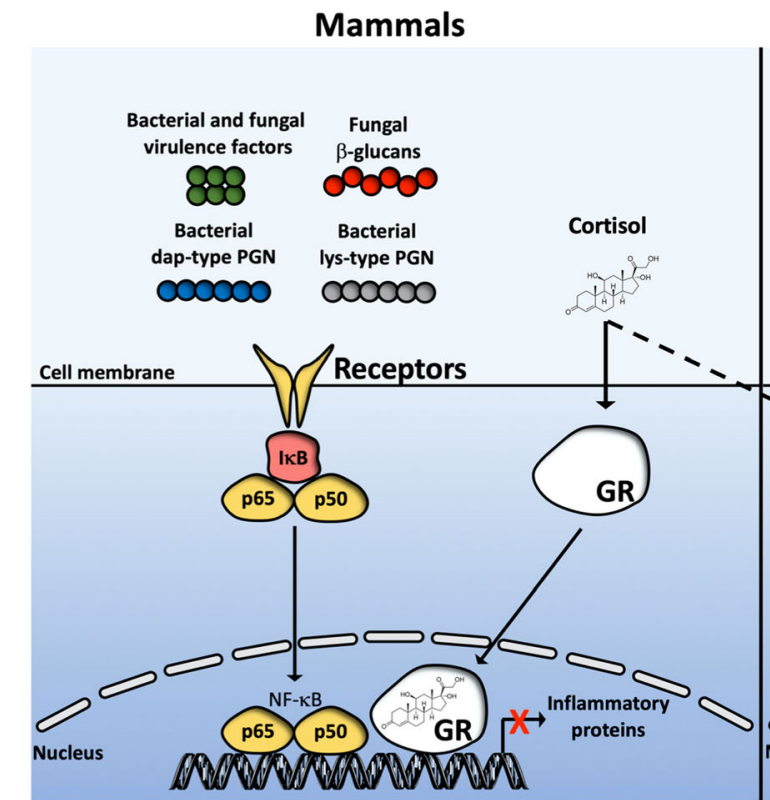

Drosophila

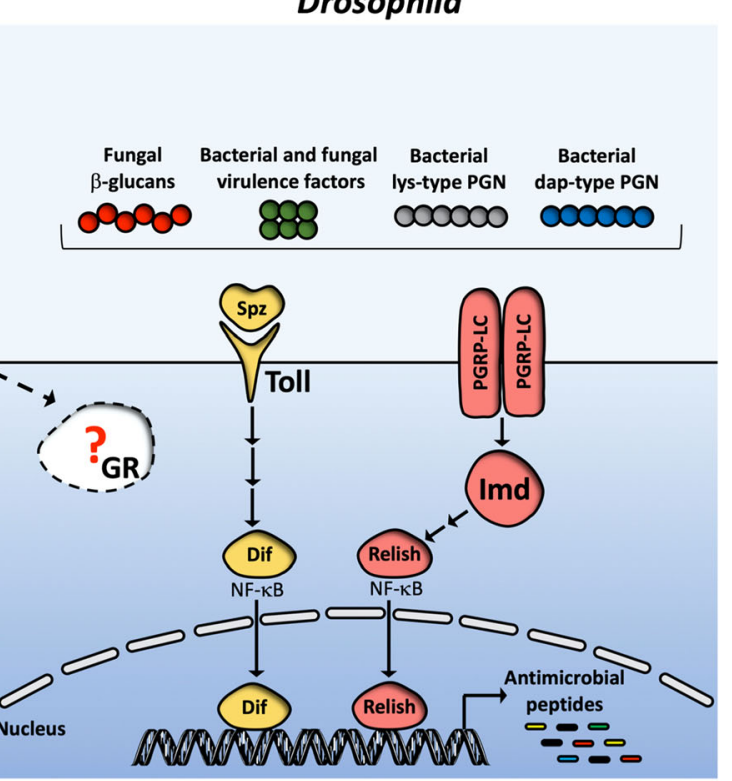

b

\begin{tabular}{|c|c|c|c|}
\cline { 2 - 4 } \multicolumn{1}{c|}{} & hsERR1 & hsER1 & hsGR \\
\hline dmERR & $44 \% / 58 \%$ & $35 \% / 55 \%$ & $32 \% / 47 \%$ \\
\hline
\end{tabular}

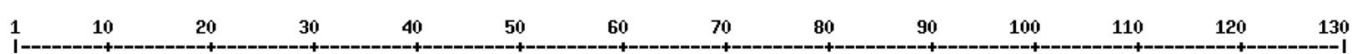

dnERR

hSGR MDSKESLTPGREENPSSYLAQERGDYMFYKTLRGGATYKYSASSPSLAYASQSDSKQQRRLLYDFPKGSYSNAQQPDLSKAYSLSMGL YHGETETKYMGNDLGFPQQGQISLSSGETDLKLLEESTANLN hsER1

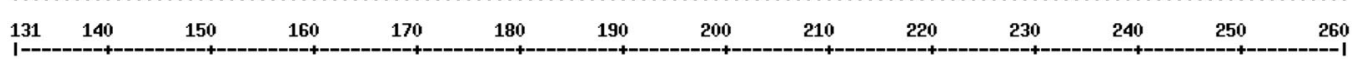
dnERR Consensus

RSTSYPENPKSSASTAYSAAPTEKEFPKTHSDYSSEQQHLKGQTGTHGGNYKLYITDQSTFDILQDLEFSSGSPGKETHESPHRSDLLIDENCLLSPLAGEDDSFLLEGNSNEDCKPLILPDTK-PKIKD

$261 \quad 270 \quad 280 \quad 290$ .................

dnERR
hsERRI

hSERR1 NGDL YLSSPSNYTLPQYETEKEDFIELCTPGYIKQEKLGTYYCQASFPGANIIGNKMSAISYHGYSTSGGQHYHYDHNTASLSQQQDQRPIFNYIPPIPYGSENHNRCQGSGDDNLTSLGTLNFPGRTYF Consensu LGEYYLDSSKPAYYNYPEGAAYEFNAFAFANAQVYGQTGLPYGPGSEAFAFGSNGLGGFPPLNSYSPSPLHLLHPPPQLSPFLQPHGQQ-------YPYYLENEPSGYTVREAGPPAFYRPNSDNRR--

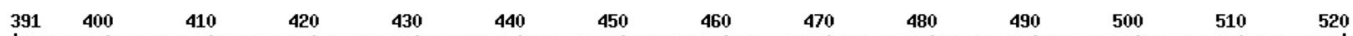

dMERR GGNGGGGGGGSGGNATHASAGAGSGSYRDEL RLCLYCGDYASGFHYGYASCEACKAFFKRTIQGNIEYTCPANNECETNKRRRKACQACRFQKCLLMGGLKEGYRLDRV :GGRQKYRRNPYSNSYQTH

hsGR SNGYSPSMPPDVSSPPSSST

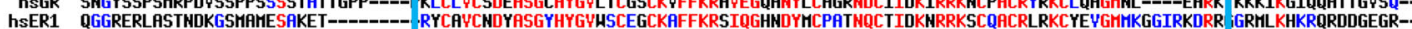

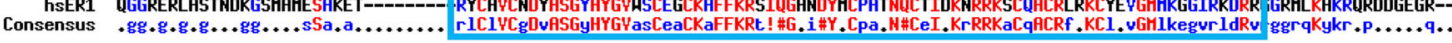

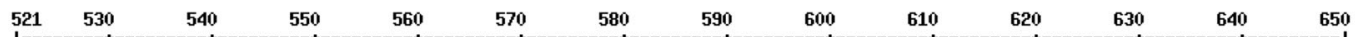
dnERR QLLYQSNTTSLCDVKILEYLNSYEPDALSYQTPPPQY ITTSITNDEASSSSGSIKLESSYYTPNGTCIFQNNNNNDPNEILSYLSDIYDKELYSYIGHAKQTPGFIDLPLNDQHKLLQYSHAEILTLQLT

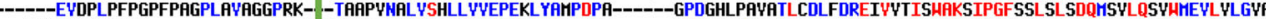
-

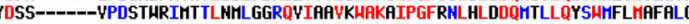

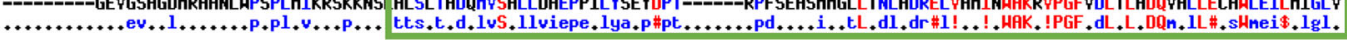

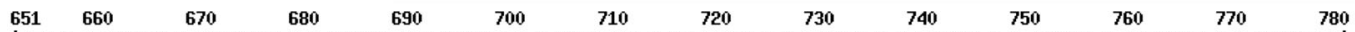
dnERR FRSLPFNGK--LCFATDYHMDEHLAKEC-GYTEFYYHCYQIAQRHERISPRREEYYLLKALLLANC-----D-ILLDDQSSLRAFRDTILNSLNDYYYLLRHSSAYSHQ--QQ---LLLLLPSLRQADDI hsERR1 QRSLPLQDE--LAFFEDLYLDEEGARAA-GLGELGAALLQL YRRL QALRLEREEYYLLKALAL ANS-----DSVHIEDAEAYEOLREALHEALLEYEAGRAGPGGGGER--RRAGRLLTLPLLROTAGK

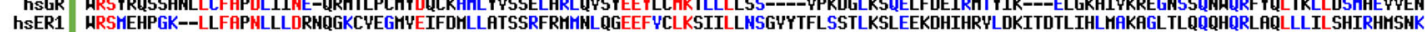

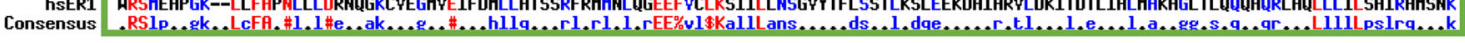

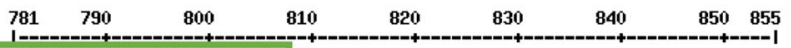

\begin{tabular}{r|l} 
dnERR & LRRFHRGIARDEVITHKKLFLEYLEPL \\
hSERR1 & YLAHFYGYKLEGKYPHHKLFLEMLEFH iD
\end{tabular}

hsGR LLNYCFQTFLOKTHSIEFPEHLFEIIT IOIPKYSNGNIKKLFFOK

hSER1 GMEHLYSMKCKNYYPLYDLLLEMLDAH LLHAPISRGGASYEETDQSHLATAGSISSHSLQKYYITGEREGFPATY

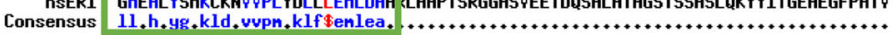

Fig. 1 (See legend on next page.) 
(See figure on previous page.)

Fig. 1 Identification of GR sequence-homolog in Drosophila melanogaster. a An illustration of the hypothesis for the existence of GR ortholog in fruit flies. During mammalian microbial infection (left panel), cell surface receptors, such as Toll-like receptors, detect bacterial and fungal cells. Once bound to microbial cells, mammalian receptors activate the expression of pro-inflammatory genes through the actions of Nuclear Factor $\mathrm{KB}$ (NF-KB). Steroids, such as cortisol, binds to a nuclear receptor, glucocorticoid receptor (GR), and trigger its translocation into the nucleus, where it represses NF-KB and the expression of pro-inflammatory genes. Similar pathways exist in insects (right panel), where two pathways detect invading microbial pathogens, and ultimately trigger the expression of antimicrobial peptides. Toll and Imd pathways both ultimately activate NFKB transcription factors, which induce the transcription of antimicrobial peptides. We hypothesize the existence of a GR ortholog in fruit flies, capable of immunosuppressing infected insects in response to steroids. b Multiple amino acid sequence alignment between D. melanogaster Estrogen Related Receptor (dmERR), Homo sapiens ERR (hsERR1), H. sapiens estrogen receptor (hsER1), and H. sapiens glucocorticoid receptor (hsGR). The sequences of dmERR (Accession NP_648183), hsERR1 (Accession XP_016872802), hsER1 (Accession XP_016865870), and hsGR (Accession CAJ65924) were aligned using MultAlin software. The DNA binding domain and ligand binding domain are highlighted in blue and green boxes, respectively. Identical amino acids are shown in red. The extent (\%) of the identity/similarity between Drosophila and human sequences is shown above the alignment

respectively). Moreover, this data demonstrated that $S$. cerevisiae was more pathogenic to flies than any of the tested bacteria, since it took three orders of magnitude less of yeast cells to achieve a comparable death rate of flies caused by bacterial pathogens. Specifically, the fly death rate caused by $8.30 \times 10^{6}$ yeast cells $/ \mathrm{ml}$ (Fig. 2a) is comparable to the fly death rate caused by $4.00 \times 10^{9}$ bacterial cells/ml (Figs. 2b-e).

While uninfected flies feeding solely on sucrose can survive for 10-14 days, some of the flies die at earlier times, such as after 100-150 h. Therefore, to study the effects of GCs on the sensitivity of Drosophila to yeast, a mid-concentration of $1.67 \times 10^{7}$ fungal cells $/ \mathrm{ml}$ in the sucrose solution was used for the remainder of the study, because it caused fly death well before $100 \mathrm{~h}$.

\section{Increased susceptibility of Drosophila to fungal infection with oral exposure to cortisone acetate}

GCs are commonly prescribed for the treatment of numerous inflammatory and autoimmune diseases, as well as used as immunosuppressants to reduce the likelihood of organ rejection in organ transplant recipients $[1,3]$. Cortisone acetate (CA), a synthetic GC converted to the naturally occurring cortisol within the human body (Fig. 3a), reduces the possibility of organ rejection while simultaneously increasing the susceptibility of recipients to fungal infections [22]. The significance of human CA-induced immunosuppression to fungal pathogens motivated us to select yeast for our subsequent tests. We tested the ability of CA to elicit a similar effect in Drosophila when orally exposed during a S. cerevisiae infection. Chamilos, et al. demonstrated increased susceptibility of wildtype flies to Rhizopus oryzae zygomycotic systemic infection when flies are orally exposed to $20 \mathrm{mg} / \mathrm{ml}(50 \mathrm{mM})$ of the synthetic GC, dexamethasone [14].

For our study, we orally provided CA to wildtype flies in conjunction with exposure to S. cerevisiae in sucrose solution. Various concentrations of $\mathrm{CA}$, ranging from $20 \mathrm{mM}$ to $140 \mathrm{mM}$, were provided to determine a CA concentration where flies of both genders were more susceptible to S. cerevisiae infection (Fig. 3b and Additional file 3). Additionally, we determined that none of the tested CA concentrations were toxic to uninfected flies. The concentrations of CA found to significantly increase the susceptibility of female and male flies to infection were $100 \mathrm{mM}$ and $140 \mathrm{mM}$, respectively. Both concentrations for each gender led to decreased time to death. In males, who required exposure to a higher concentration of CA than females to increase susceptibility to $S$. cerevisiae, the median survival was reached $20 \mathrm{~h}$ sooner with CA compared to the control without CA. Females exhibited a similar effect on median survival when exposed to $100 \mathrm{mM}$ CA: median survival was shortened by $25 \mathrm{~h}$. These results show that, like in mammalian species, cortisone acetate increased the susceptibility of flies to fungi.

\section{Increased susceptibility of flies to fungal infection with oral exposure to mammalian sex hormone}

We sought to test whether other known steroid ligands of human NR subfamily 3 could also increase Drosophila susceptibility to $S$. cerevisiae infection. Because dmERR is homologous to both hsERR1 and hsER1, we tested the ability of a mammalian ER ligand, the estrogen steroid hormone, 17ß-estradiol (17ß-E) (Fig. 3a), to affect fly susceptibility to $S$. cerevisiae infection. 17ß-E, a molecule with both anti- and pro-inflammatory effects [23], exhibits a steroid skeleton structure very similar to CA.

Since $17 ß-E$ is a female hormone in mammals, we utilized only female flies to determine the effect of the compound on the susceptibility of flies to S. cerevisiae infection. $17 ß-E$ was orally supplied within the concentration range as that of CA. At $75 \mathrm{mM}, 17 ß-\mathrm{E}$ was more effective than CA in increasing the susceptibility of female wildtype flies to $S$. cerevisiae infection, without causing toxicity to uninfected flies (Fig. 3c). The median survival of infected flies occurred $15 \mathrm{~h}$ sooner in the presence of $17 ß-E$ than without this compound. This data demonstrates that multiple steroid hormones could increase the sensitivity of flies to fungal challenge. 
a

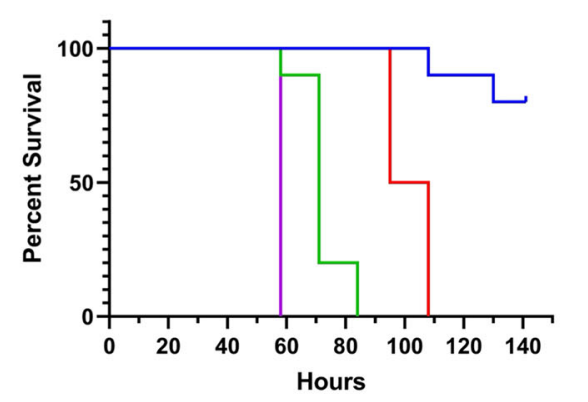

b

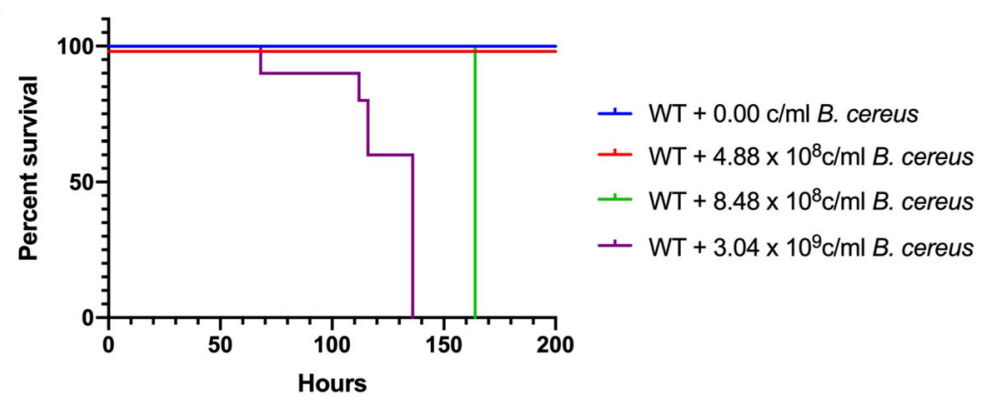

C

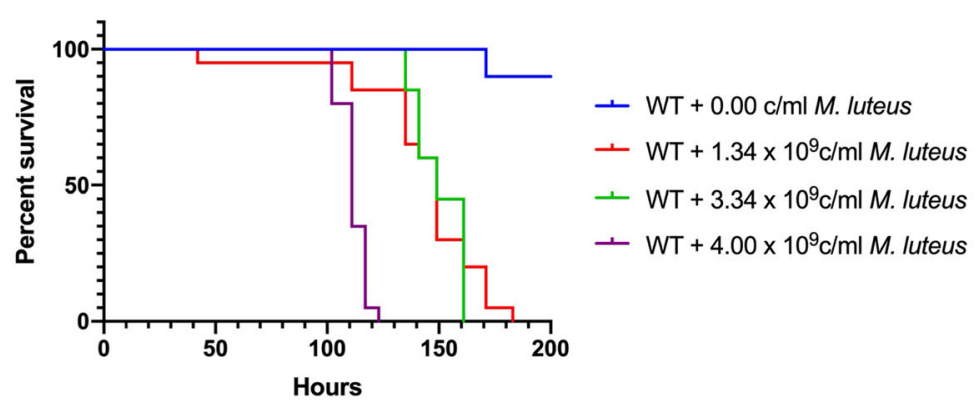

d

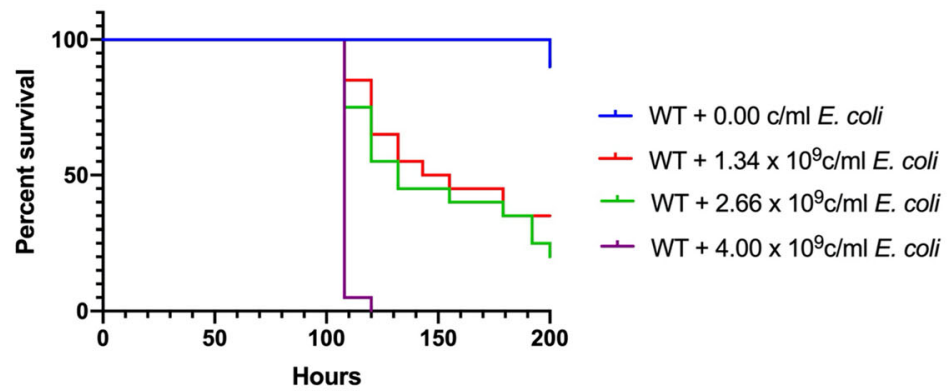

e

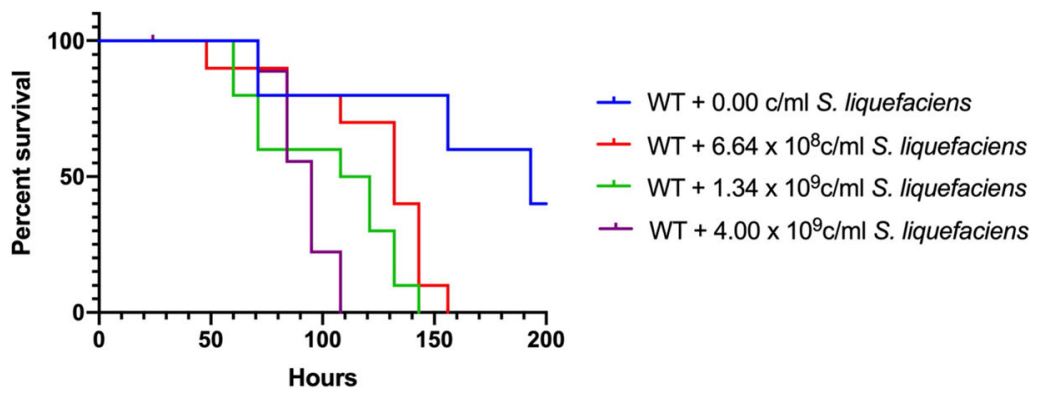

- WT $+0.00 \mathrm{c} / \mathrm{ml} \mathrm{S}$. cerevisiae

- WT $+8.30 \times 10^{6} \mathrm{c} / \mathrm{ml}$ S. cerevisiae

- WT $+1.67 \times 10^{7} \mathrm{c} / \mathrm{ml} \mathrm{S}$. cerevisiae

$+\mathrm{WT}+4.17 \times 10^{7} \mathrm{c} / \mathrm{ml} \mathrm{S}$. cerevisiae
- WT $+4.88 \times 10^{8} \mathrm{c} / \mathrm{ml} \mathrm{B}$. cereus

- WT $+8.48 \times 10^{8} \mathrm{c} / \mathrm{ml}$ B. cereus

- WT $+3.04 \times 10^{9} \mathrm{c} / \mathrm{ml} \mathrm{B}$. cereus
- WT $+1.34 \times 10^{9} \mathrm{c} / \mathrm{ml} M$. luteus

- WT $+3.34 \times 10^{9} \mathrm{c} / \mathrm{ml}$ M. luteus

- WT $+4.00 \times 10^{9} \mathrm{c} / \mathrm{ml}$ M. luteus

- WT $+1.34 \times 10^{9} \mathrm{c} / \mathrm{ml}$ E. coli

- WT $+2.66 \times 10^{9} \mathrm{c} / \mathrm{ml}$ E. coli

- WT $+4.00 \times 10^{9} \mathrm{c} / \mathrm{ml} \mathrm{E}$. coli

Fig. 2 (See legend on next page.) 
(See figure on previous page.)

Fig. 2 Sensitivity of female wildtype flies to microbial infections. Female Oregon-R wild type (WT) flies were orally challenged with different amounts of (a) S. cerevisiae, (b) Bacillus cereus, (c) Micrococcus luteus, (d) Escherichia coli, and (e) Serratia liquefaciens. Flies were fed in vials with 50 $\mathrm{mM}$ sucrose solution containing various microbial concentrations. Each condition contains ten flies. Vials are incubated at $30^{\circ} \mathrm{C}$ and checked a minimum of twice per day for fly survival

\section{Effect of estrogen on Culex quinquefasciatus mosquitoes during fungal infection}

To determine whether steroids affect the sensitivity of other insects to yeast infections, we searched for ERR homologs in other species. The genome of Culex quinquefascuiatus mosquitoes harbors a close homolog of fly $E R R$, with $63 \%$ amino acid identity and $75 \%$ similarity (Fig. 4a). The function of C. quinquefascuiatus ERR homolog, locus EDS37237, is currently unknown and annotated as $E R R$ based on its sequence similarity to other ERR genes. Because mosquitoes contain an ERR ortholog, we tested the ability of a steroid molecule to affect the sensitivity of yeast-infected C. quinquefascuiatus, who just like Drosophila, is a member of the insect order Diptera [24].

Before the exposure to $17 \beta-\mathrm{E}$, we determined $3.3 \times$ $10^{7}$ fungal cells $/ \mathrm{ml}$ as the minimal lethal dose necessary to kill female adult $C$. quinquefascuiatus mosquitoes, aged 4-5 days. Female mosquitoes were then orally exposed to $17 \AA-E$, the same concentration shown to immunosuppress flies (Fig. 4b). Like fly experiments, we observed that $75 \mathrm{mM}$ of $17 ß-\mathrm{E}$ a

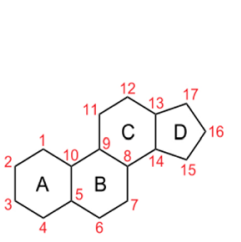

Steroid skeleton

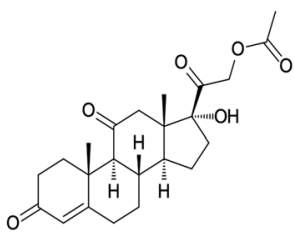

Cortisone Acetate

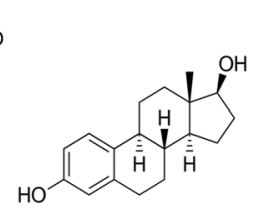

$17 ß$ - estradiol<smiles>CC(C)CC=CC(C)[C@H]1CCC2C3=CC=C4CC(O)CCC4(C)C3CCC21C</smiles>

Ergosterol

b

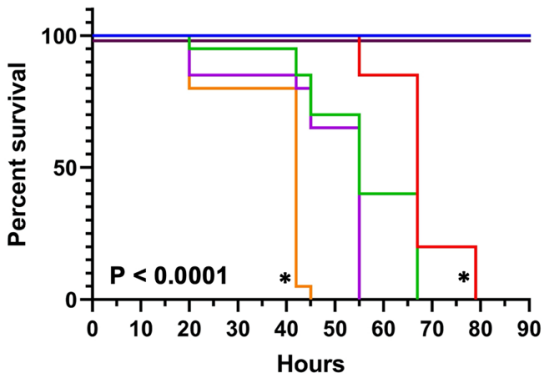

$$
\begin{aligned}
& -W T \\
& -W T+\text { S. cerevisiae } \\
& -W T+\text { S. cerevisiae + } 20 \mathrm{mM} \mathrm{CA} \\
& -W T+\text { S. cerevisiae }+60 \mathrm{mM} \mathrm{CA} \\
& -W T+\text { S. cerevisiae }+100 \mathrm{mM} \mathrm{CA} \\
& -W T+100 \mathrm{mM} \mathrm{CA}
\end{aligned}
$$

c

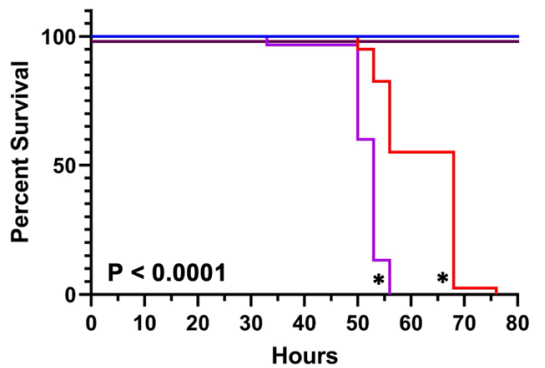

$$
\begin{aligned}
& -W T \\
& -W T+\text { S. cerevisiae } \\
& -W T+\text { S. cerevisiae + } 75 \text { mM 17ß-E } \\
& -W T+75 \text { mM 17ß-E }
\end{aligned}
$$

Fig. 3 Steroid molecules increase the sensitivity of female wildtype flies to Saccharomyces cerevisiae. a Structures of steroid skeleton and cortisol analogs tested in this study: cortisone acetate, 17ß-estradiol, and ergosterol. (b-c) Female wild type (WT) flies were orally challenged as in Fig. 1 with $1.67 \times 10^{7} \mathrm{~S}$. cerevisiae cells/ml with and without various concentrations of cortisone acetate (CA) (b) and 17ß-estradiol (17ß-E) (c). Uninfected flies exposed to $100 \mathrm{mM}$ cortisone acetate or $75 \mathrm{mM}$ 17ß-estradiol were included to test the toxicity of these compounds. P-values in b-c indicate statistical significance compared to the yeast-only condition (asterisks) on the basis of the Log-rank (Mantel-Cox) test 


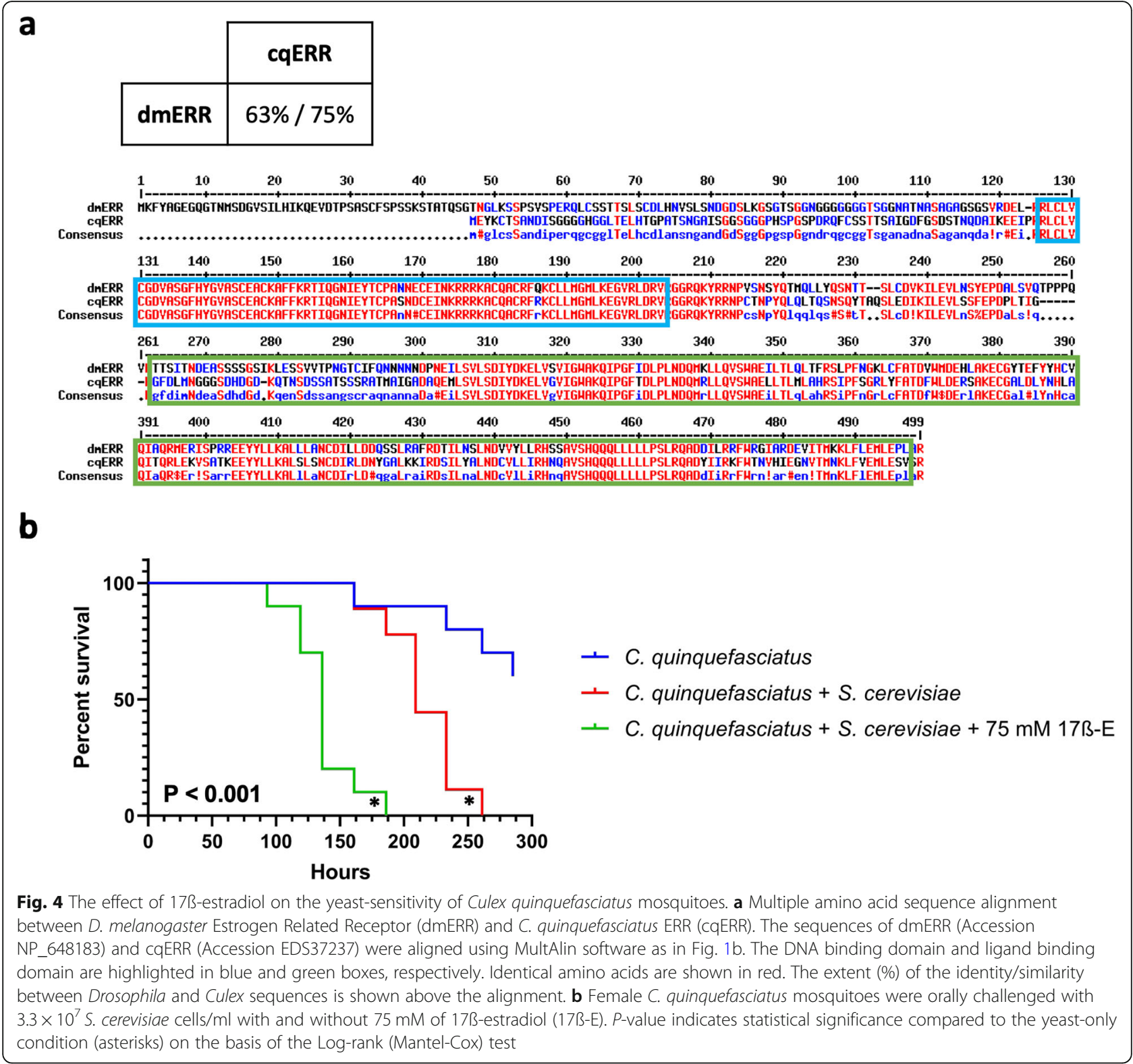

increased the sensitivity of mosquitoes to $S$. cerevisiae and decreased median survival time by $73 \mathrm{~h}$. This data shows the steroid hormone-mediated increased sensitivity to fungal infection is conserved in multiple insect species harboring the ERR gene.

\section{Drosophila ERR is necessary for CA- and 17ß-E-mediated increased sensitivity to fungal infection}

To determine whether dmERR acts as a functional human GR ortholog, female ERR homozygous lossof-function mutant flies were challenged with $S$. cerevisiae in the presence or absence of CA (Fig. 5a) or $17 ß-E$ (Fig. 5b). We observed that in the absence of steroids, ERR mutant flies exhibited similar sensitivity to yeast infection as wildtype Drosophila (median survival at 50-70 h) (Fig. 5), allowing for a parallel analysis between both fly strains. Interestingly, with the oral exposure of CA or 17ß-E, dmERR mutant flies did not show increased susceptibility to $S$. cerevisiae infection at concentrations found to increase the susceptibility of wildtype flies $(100 \mathrm{mM}$ for CA and $75 \mathrm{mM}$ for $17 ß-E)$. The median survival time of dmERR mutant flies exposed to S. cerevisiae + CA or S. cerevisiae $+17 \AA-E$ was comparable to the median survival time of wildtype flies to $S$. cerevisiae alone, which illustrated the inability of dmERR mutant flies to become more susceptible to a fungal infection in the presence of CA or $17 ß-E$. This data showed that Drosophila ERR is necessary for steroid-mediated increased sensitivity of flies to fungal infection. 


\section{a}

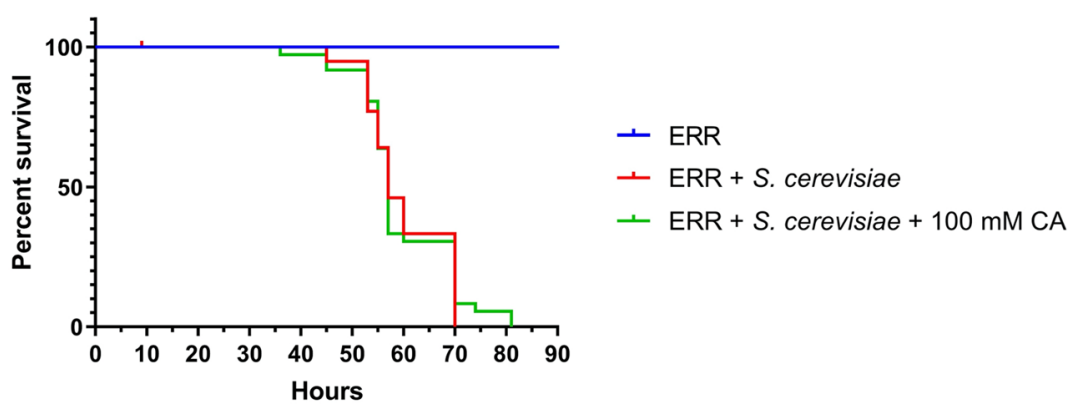

b

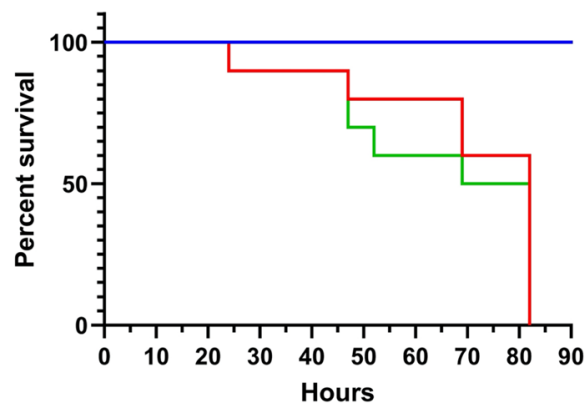

- ERR

- ERR + S. cerevisiae

- ERR + S. cerevisiae+ $75 \mathrm{mM} 17 ß-\mathrm{E}$

Fig. 5 The yeast-sensitivity of ERR mutant flies is unaffected by steroid molecules. Female Estrogen Related Receptor (ERR) mutant flies were orally challenged as in Fig. 3 with $1.67 \times 10^{7}$ S. cerevisiae cells $/ \mathrm{ml}$ with and without $100 \mathrm{mM}$ cortisone acetate (a) and $75 \mathrm{mM} 17 \mathrm{~B}$-estradiol (b)

\section{Determination of the effect of fungal ergosterol on the susceptibility of Drosophila to yeast infection}

While CA and 17ß-E are naturally occurring soluble and plasma-circulating mammalian steroids $[3,25]$, we investigated the effect of fungal steroids on the sensitivity of flies to yeast. Previously, S. cerevisiae has been shown not to generate extracellular soluble steroids [26] and instead only produces cell membrane-bound steroid-like ergosterol, which is important for maintaining membrane fluidity, permeability, and structure [27]. Like CA and $17 ß-E$, ergosterol exhibits the steroid skeleton but with a longer side chain on the 17th carbon atom (Fig. 3a). We explored the hypothesis that after ingestion of S. cerevisiae cells, fungal ergosterol may affect the sensitivity of Drosophila to S. cerevisiae. Ergosterol was exogenously provided to female wildtype flies at a range from 100 to $200 \mathrm{mM}$ (Fig. 6). At concentrations tested previously for CA and $17 ß-E$, ergosterol yielded no increased susceptibility of flies to $S$. cerevisiae infection (Fig. 6a). Even at double the concentration (200 mM), no effect on fly sensitivity was also seen (Fig. 6b).

\section{Discussion}

Here, we presented dmERR is required for steroidmediated increased fly sensitivity to fungal infections. Previous studies have reported roles for dmERR in carbohydrate metabolism and hypoxic responses [28-30]. Other research has focused on the possible role of dmERR in mitochondrial biogenesis [31], as hsERR1 has been shown to have a role in generating mitochondria [32]. In humans, the expression of medium-chain acyl coenzyme A dehydrogenase (MCAD), which is an enzyme that mediates the mitochondrial beta-oxidation of fat, is regulated by an ERR- $\alpha$ response element (ERRE) present in the $5^{\prime}$-flanking region. In COS-7 cells, hsERR1 interacts with the MCAD nuclear receptor response element 1 (NRRE-1). hsERR1 may regulate cellular energy balance by controlling the expression of MCAD through the NRRE1 [33]. Recent studies demonstrated that mitochondria are critical in stimulating innate immune signaling. Specifically, released mitochondrial DNA (mtDNA) and mitochondria-derived reactive oxygen species (mtROS) activate innate immune responses, such as inflammasome, sGAS-STING, and NF$\kappa B$ signaling pathways [34]. In addition, changes in mitochondria and metabolic pathways (TCA cycle, oxidative phosphorylation, and fatty acid oxidation) induce transcription in immune cells. For example, M1 macrophages with an impaired TCA cycle have a pro-inflammatory response, and M2 macrophages perform $\beta$-oxidation to produce anti-inflammatory responses [35]. Additionally, mitochondria are known to induce the inflammatory response: mitochondrial antiviral signaling and NLRP3 can be activated by mitochondria [36]. Moreover, the mass and mobility of mitochondria are affected by fission and 
a

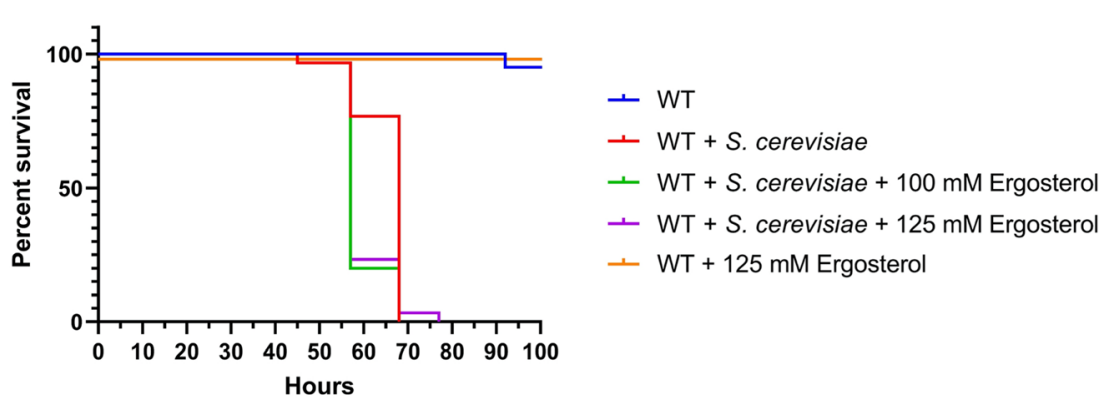

$\begin{array}{ll} & \text { WT } \\ - & W T+\text { S. cerevisiae }\end{array}$

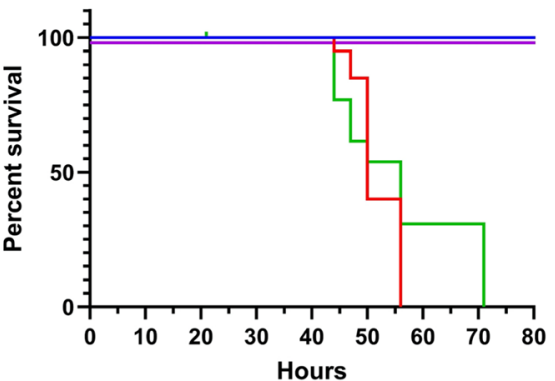

$+W T+$ S. cerevisiae

- WT + S. cerevisiae + $200 \mathrm{mM}$ Ergosterol

$-W T+200 \mathrm{mM}$ Ergosterol

Fig. 6 Fungal ergosterol does not affect the yeast-sensitivity of female wildtype flies. Female wild type (WT) flies were orally challenged as in Fig. 3 with $1.67 \times 10^{7}$ S. cerevisiae cells $/ \mathrm{ml}$ with and without indicated concentrations of ergosterol

fusion that affect the immune functions [37]. In immune cells, mitochondria are located close to the endoplasmic reticulum (ER), which allows cells to control metabolism that is essential for immune functions [37]. Our results are the first to allude the role of dmERR in Drosophila immunity, specifically in increasing the susceptibility of flies to fungal infections when orally exposed to steroid molecules. Specifically, we have shown the ability of synthetic $\mathrm{GC}, \mathrm{CA}$, to increase the susceptibility of flies to fungal infection when orally presented. This is consistent with dmERR being the only homolog for members of the human NR subfamily 3, in which GR is included. dmERR is necessary for steroid-induced immunosuppression of flies and is highly homologous to GR. Thus, we propose that $\mathrm{dmERR}$ is the functional ortholog of GR.

Not all molecules exhibiting the steroid backbone elicit the same effect on fly sensitivity to fungal infection. Tested concentrations of ergosterol did not increase fly sensitivity to S. cerevisiae infection. Unlike CA and $17 ß-$ $\mathrm{E}$, which are transported to their target via the bloodstream in mammals, ergosterol is not soluble and/or mobile. Rather, it is integrated within the cell wall of $S$. cerevisiae and may not be readily bioavailable for fly ingestion. It is possible ergosterol elicits an effect at a higher concentration than tested, but such concentration may not be physiologically relevant. The inability of ergosterol to sensitize flies to $S$. cerevisiae could be either because ergosterol is not a ligand for dmERR or because the supplied ergosterol is soluble and out of the context of the fungal membrane.

Similarly to human ERR, the ligand for dmERR has yet to be discovered. Several studies have alluded to the existence of a ligand for dmERR [13, 38, 39], but a definitive ligand has not been reported. If $\mathrm{dmERR}$ is indeed the functional ortholog of human GR, there exists a greater possibility of the existence of a natural ligand for dmERR. If one is found to exist, Drosophila may be used as a model to identify a ligand for hsERR1 in humans, relieving the NR of its orphan status and providing a deeper understanding of the biological functions of dmERR and/or hsERR1. Additionally, D. melanogaster's sophisticated innate immune system has largely evolved to combat bacterial and fungal pathogens relevant to the understanding of human inflammatory conditions [40]. In response to pathogenic challenges, AMPs are released through two primary pathways that involve evolutionarily conserved components, including Toll and Toll-like receptors, as well as NF- $\mathrm{BB}$, tumor necrosis factor- $\alpha$, and JAK/STAT signaling [41]. Thus, human mutations identified in hsERR1 can be studied by creating humanized dmERR to elucidate the innate immune effects of those genetic changes, as Drosophila lack adaptive immunity.

Fruit flies have thus far only one identified steroid hormone, 20-hydroxyecdysone (20E). Ecdysone was shown to regulate both immunity and major developmental transitions in the fly, such as metamorphosis [42]. Two 
Drosophila nuclear receptors are known to be receptors for 20E: ecdysone receptor (EcR) and ultraspiracle (USP). EcR binds to 20E, heterodimerizes with USP [43], and activates the expression of a large set of genes known to function in cell motility, cell shape, and phagocytosis [42]. Ecdysone-regulation in Drosophila was shown to be essential for hemocyte immune functions and survival after infection: 20E induces the phagocytosis in Drosophila hemocytes, and larvae lacking ecdysone-activated hemocytes are defective in bacterial phagocytosis and are susceptible to oral bacterial infections. In contrast, the results presented here show that the role of Drosophila ERR is to suppress the immunity of flies, which may be needed to counterbalance the positive effect of EcR on immunity.

While this study observes the necessity of fly ERR for the steroid-mediated immunosuppression, future studies will focus on the mechanism by which GC affects ERR, such as whether GC binds to ERR and induces its nuclear translocation, followed by its nuclear activity. Mammalian GRs are known to affect the immunity by two nuclear mechanisms: by binding directly to and repressing the transcriptional activity of NF- $\mathrm{KB}$, as well as by binding directly to the promoters of immunityrelated genes and regulating their transcription. Moreover, GRs affect immunity-related cytoplasmic proteins by a third non-nuclear mechanism via activating cytoplasmic phosphatidylinositol 3-kinase and protein kinase Akt, leading to the activation of secondary messengers nitric oxide [3]. Investigating whether fly ERR is affecting immunity-related genes and processes analogously to the three mammalian mechanisms will help us understand how GC influences ERR and innate immunity pathways.

\section{Conclusions}

This study identifies a D. melanogaster gene that structurally resembles vertebrate GR and is functionally necessary for the steroid-mediated immunosuppression to fungal infections.

\section{Methods}

\section{Drosophila rearing}

Drosophila melanogaster strains were housed at $25^{\circ} \mathrm{C}$ with 12-h light/dark cycles and fed on standard cornmeal-molasses-agar fly medium with yeast flakes. Wildtype experiments were conducted with Oregon-R, selected for their rapid egg-laying ability (Bloomington Drosophila Stock Center (BDSC) stock \#2376), Drosophila aged 4-5 days. Experiments with homozygous ERR (BDSC stock \#28467) Drosophila utilized unaged flies at the time of the experiments.

\section{Drosophila oral feeding survival assay}

Saccharomyces cerevisiae diploid strain YEF473, ATCC ${ }^{\bullet}$ 200970 [44] was used as the infective agent for all Drosophila survival assays. S. cerevisiae was incubated on YPD medium at $30^{\circ} \mathrm{C}$. Overnight cultures were grown in YPD at $30^{\circ} \mathrm{C}$ at $180 \mathrm{rpm}$ for $14-16 \mathrm{~h}$.

Bacillus cereus (ATCC 10987), Escherichia coli (C600), Micrococcus luteus (ATCC 4698), and Serratia liquefaciens (ATCC 27592) were used. B. cereus and E. coli were cultured in Lysogeny broth (LB) at $37^{\circ} \mathrm{C}$. M. luteus was grown in $\mathrm{LB}$ at $25^{\circ} \mathrm{C}$. S. liquefaciens was incubated in Tryptic Soy Broth (TSB) at $30^{\circ} \mathrm{C}$ overnight.

Flies were infected according to the microbial intestinal infection methods described previously in Nehme, et al [45] with the following modifications. Drosophila vials were prepared by placing three $25 \mathrm{~mm}$ diameter circles of extra-thick Whatman blotting paper (Bio-Rad Laboratories, catalog \#1703965) at the bottom of the vials and capping with a foam plug. S. cerevisiae overnight cultures were centrifuged, and the pellets resuspended in $50 \mathrm{mM}$ sucrose solution to a final desired optical density ( 0.83 $\mathrm{OD}, 1.7 \mathrm{OD}$ or $3.3 \mathrm{OD})$ at $600 \mathrm{~nm}\left(\mathrm{OD}_{600}\right)$. $\mathrm{OD}_{600}$ values were converted to cells/ml $\left(\mathrm{OD}_{600}\right.$ of 1.0 corresponds to approximately $10^{7}$ cells $/ \mathrm{ml}$ ) [46]. Bacterial infections were carried out analogously, except at higher cells/ml (converted from $\mathrm{OD}_{600}$ values according to McFarland's scale) $[47,48]$. Depending on the experiment, steroid molecules were added to the fungal sucrose solution. 17ß-estradiol (catalog \#10006315), cortisone acetate (catalog \#23798), and ergosterol (catalog \#19850) were all purchased from the Cayman Chemical Company. Each prepared Drosophila vial contained $2.5 \mathrm{ml}$ of its respective solution, which was absorbed by the Whatman paper found at the bottom of the vial. Flies were anesthetized by $\mathrm{CO}_{2}$, separated by gender, and placed into the Drosophila vials, with ten flies in each vial. Vials were incubated at $30^{\circ} \mathrm{C}$ and checked a minimum of twice per day for fly survival.

\section{Mosquito rearing}

C. quinquefasciatus mosquitoes were obtained from a colony maintained by Benzon Research (Carlisle, PA, USA). Mosquitoes were reared and maintained at $28^{\circ} \mathrm{C}$ and $80 \%$ relative humidity in $30 \times 30 \times 30$-cm cages with 12-h light/dark cycles. Adult mosquitoes were maintained on $10 \%$ sucrose ad libitum, while larvae were fed a 1:1:1 mixture of bovine liver powder (Carlisle, PA, USA). For experiments, adult female mosquitoes aged 4-5 days were used.

\section{Mosquito oral infection survival assay}

S. cerevisiae diploid strain YEF473, ATCC 200,970 [44] was used as the infective agent for all $C$. quinquefasciatus survival assays. 
C. quinquefasciatus were infected similar to the technique used to infect Drosophila, as described above, but include the following modifications. C. quinquefasciatus vials were prepared by placing $5 \times 5-\mathrm{cm}$ of extra-thick Whatman blotting paper (Bio-Rad Laboratories, catalog \#1703965) in square-bottom, polypropylene Drosophila bottles and capped with a foam plug. S. cerevisiae overnight cultures were centrifuged, and the pellets resuspended in $50 \mathrm{mM}$ sucrose solution to a final optical density at $600 \mathrm{~nm}\left(\mathrm{OD}_{600}\right)$ of $3.3 \mathrm{OD}$. $\mathrm{OD}_{600}$ values were converted to cells $/ \mathrm{ml}\left(\mathrm{OD}_{600}\right.$ of 1.0 corresponds to approximately $10^{7}$ cells $/ \mathrm{ml}$ ) [46]. 17ß-estradiol (catalog \#10006315) steroid molecules, purchased from Cayman Chemical Company, were added to the fungal sucrose solutions. Each prepared C. quinquefasciatus bottle contained $10 \mathrm{ml}$ of its respective solution and absorbed by the Whatman paper found at the bottom of each vial. Mosquitoes were anesthetized using $\mathrm{CO}_{2}$, separated by gender, and placed in the prepared vials, with ten mosquitoes in each vial. Vials were incubated at $30^{\circ} \mathrm{C}$ and checked a minimum of once a day for mosquito survival.

\section{Supplementary information}

Supplementary information accompanies this paper at https://doi.org/10. 1186/s12866-020-01848-X.

Additional file 1. The amino acid sequence similarity between DNA and ligand binding domains of dmERR with hsERR1, hsER1, and hsGR. The amino acid sequences of DNA binding domain (DBD) and ligand binding domain (LBD) are compared between dmERR, hsERR1, hsER1, and hsGR. The extent (\%) of the identity/similarity is shown for each domain.

Additional file 2. Sensitivity of male wildtype flies to Saccharomyces cerevisiae infection. Male Oregon-R wild type (WT) flies were orally challenged with different amounts of $\mathrm{S}$. cerevisiae. Flies were fed in vials with $50 \mathrm{mM}$ sucrose solution containing various yeast concentrations. Each condition contains ten flies. Vials are incubated at $30^{\circ} \mathrm{C}$ and checked a minimum of twice per day for fly survival.

Additional file 3. Cortisone acetate increases the sensitivity of male wildtype flies to Saccharomyces cerevisiae. Male wild type (WT) flies were orally challenged as in Fig. 1 with $1.67 \times 10^{7} \mathrm{~S}$. cerevisiae cells $/ \mathrm{ml}$ with and without various concentrations of cortisone acetate (CA). Uninfected flies exposed to $140 \mathrm{mM}$ cortisone acetate were included to test the toxicity of this compound. P-value indicates statistical significance compared to the yeast-only condition (asterisks) on the basis of the Log-rank (Mantel(ox) test.

\section{Abbreviations}

GC: Glucocorticoid; LBD: Ligand binding domain; GR: Glucocorticoid receptor; DBD: DNA binding domain; GRE: Glucocorticoid responsive elements; NFKB: Nuclear factor kB; AMP: Anti-microbial peptide; NR: Nuclear Receptor; hsERR1: Human estrogen-related receptor 1; hsER1: Human estrogen receptor 1; dmERR: Drosophila estrogen-related receptor; CA: Cortisone acetate; 17ß-E: 17ß-estradiol

\section{Acknowledgments}

Not applicable

\section{Authors' contributions}

M.M.S. designed research; G.B., L.O.G., S.A., performed research; all analyzed data; and G.B., C.A.V., and M.M.S. wrote the paper, which was edited by all authors. All authors have read and approved the manuscript.

\section{Funding}

M.M.S. acknowledges support from City of Hope Comprehensive Cancer Center through the KL2 Mentored Career Development Award Program of the Inland California Translational Consortium (GR720001).

\section{Availability of data and materials}

All data generated or analyzed during this study are included in this published article [and its supplementary information files].

Ethics approval and consent to participate

Not applicable.

\section{Consent for publication}

Not applicable.

\section{Competing interests}

The authors declare that they have no competing interests.

\section{Author details}

${ }^{1}$ Henry E. Riggs School of Applied Life Sciences, Keck Graduate Institute, Claremont, CA 91711, USA. ${ }^{2}$ Aperiomics, Inc., Sterling, VA 20166, USA. ${ }^{3}$ Lake Erie College of Osteopathic Medicine, 1858 W Grandview Blvd, Erie, PA 16509, USA. ${ }^{4}$ Department of Geriatrics, West China Hospital, Sichuan University, Chengdu, Sichuan, China.

Received: 9 March 2020 Accepted: 9 June 2020

Published online: 15 June 2020

References

1. Ramamoorthy S, Cidlowski JA. Corticosteroids. Rheum Dis Clin N Am. 2016; 42:15-31.

2. Escoter-Torres L, Caratti G, Mechtidou A, Tuckermann J, Uhlenhaut NH, Vettorazzi S. Fighting the fire: mechanisms of inflammatory gene regulation by the glucocorticoid receptor. Front Immunol. 2019;10. https://doi.org/10. 3389/fimmu.2019.01859.

3. Rhen T, Cidlowski JA. Antiinflammatory action of glucocorticoids - new mechanisms for old drugs. N Engl J Med. 2005;353:1711-23.

4. Cain DW, Cidlowski JA. Immune regulation by glucocorticoids. Nat Rev Immunol. 2017;17:233-47.

5. Buchon N, Silverman N, Cherry S. Immunity in Drosophila melanogaster from microbial recognition to whole-organism physiology. Nat Rev Immunol. 2014;14:796-810.

6. Troha K, Buchon N. Methods for the study of innate immunity in Drosophila melanogaster. Wiley Interdiscip Rev Dev Biol. 2019;8:e344.

7. Vaz F, Kounatidis I, Covas G, Parton RM, Harkiolaki M, Davis I, et al. Accessibility to Peptidoglycan Is Important for the Recognition of GramPositive Bacteria in Drosophila. Cell Reports. 2019:27:2480-2492.e6.

8. King-Jones K, Thummel CS. Nuclear receptors - a perspective from Drosophila. Nat Rev Genet. 2005;6:311-23.

9. Sever R, Glass CK. Signaling by nuclear receptors. Cold Spring Harb Perspect Biol. 2013;5:a016709.

10. A Unified Nomenclature System for the Nuclear Receptor Superfamily. Cell. 1999;97:161-3.

11. Bodofsky S, Koitz F, Wightman B. Conserved and Exapted Functions of Nuclear Receptors in Animal Development. Nuclear Receptor Research. 2017;4. doi:https://doi.org/10.11131/2017/101305.

12. Henrich VC, Beatty JM. Chapter 248 - nuclear receptors in Drosophila melanogaster. In: Bradshaw RA, Dennis EA, editors. Handbook of cell signaling (Second Edition). San Diego: Academic Press; 2010. p. 2027-37. doi:https://doi.org/10.1016/B978-0-12-374145-5.00248-5.

13. Palanker L. Dynamic regulation of Drosophila nuclear receptor activity in vivo. Development. 2006;133:3549-62.

14. Chamilos G, Lewis RE, Hu J, Xiao L, Zal T, Gilliet M, et al. Drosophila melanogaster as a model host to dissect the immunopathogenesis of zygomycosis. Proc Natl Acad Sci. 2008;105:9367-72.

15. Maglich JM, Sluder A, Guan X, Shi Y, McKee DD, Carrick K, et al. Comparison of complete nuclear receptor sets from the human, Caenorhabditis elegans and Drosophila genomes. :7.

16. Mazaira Gl, Zgajnar NR, Lotufo CM, Daneri-Becerra C, Sivils JC, Soto OB, et al. The Nuclear Receptor Field: A Historical Overview and Future Challenges. 
Nuclear Receptor Research. 2018;5. doi:https://doi.org/10.11131/2018/ 101320

17. Mondotte $\mathrm{JA}$, Saleh M-C. Antiviral immune response and the route of infection in Drosophila melanogaster. In: Advances in Virus Research. Elsevier; 2018. p. 247-78. https://doi.org/10.1016/bs.aivir.2017.10.006.

18. Madden AA, Epps MJ, Fukami T, Irwin RE, Sheppard J, Sorger DM, et al. The ecology of insect-yeast relationships and its relevance to human industry. Proc R Soc B Biol Sci. 2018;285:20172733.

19. Quan AS, Eisen MB. The ecology of the Drosophila-yeast mutualism in wineries. PLoS One. 2018;13:e0196440.

20. Nehme NT, Quintin J, Cho JH, Lee J, Lafarge M-C, Kocks C, et al. Relative roles of the cellular and Humoral responses in the Drosophila host defense against three gram-positive bacterial infections. PLoS One. 2011;6:e14743.

21. Tracy C, Krämer H. Escherichia coli Infection of Drosophila. Bio Protoc. 2017;7. doi:https://doi.org/10.21769/BioProtoc.2256.

22. Corticosteroids. In: LiverTox: Clinical and Research Information on DrugInduced Liver Injury. Bethesda (MD): National Institute of Diabetes and Digestive and Kidney Diseases; 2012. http://www.ncbi.nlm.nih.gov/books/ NBK548400/. Accessed 11 Nov 2019.

23. Bereshchenko O, Bruscoli S, Riccardi C. Glucocorticoids, sex hormones, and immunity. Front Immunol. 2018:9:1332.

24. Arensburger P, Megy K, Waterhouse RM, Abrudan J, Amedeo P, Antelo B, et al. Sequencing of Culex quinquefasciatus establishes a platform for mosquito comparative genomics. Science. 2010;330:86-8.

25. Jacobs* MN, Lewis DFV. Steroid hormone receptors and dietary ligands: a selected review. Proc Nutr Soc. 2002;61:105-22.

26. Feldman D, Krishnan A. Estrogens in unexpected places: possible implications for researchers and consumers. Environ Health Perspect. 1995; 103:5.

27. Bhattacharya S, Esquivel BD, White TC. Overexpression or Deletion of Ergosterol Biosynthesis Genes Alters Doubling Time, Response to Stress Agents, and Drug Susceptibility in Saccharomyces cerevisiae. mBio. 2018;9: e01291-18, /mbio/9/4/mBio.01291-18.atom.

28. Li Y, Padmanabha D, Gentile LB, Dumur Cl, Beckstead RB, Baker KD. HIF- and non-HIF-regulated hypoxic responses require the estrogen-related receptor in Drosophila melanogaster. PLoS Genet. 2013;9:e1003230.

29. Li H, Chawla G, Hurlburt AJ, Sterrett MC, Zaslaver O, Cox J, et al. Drosophila larvae synthesize the putative oncometabolite L-2-hydroxyglutarate during normal developmental growth. Proc Natl Acad Sci U S A. 2017;114:1353-8.

30. Tennessen JM, Baker KD, Lam G, Evans J, Thummel CS. The Drosophila estrogen-related receptor directs a metabolic switch that supports developmental growth. Cell Metab. 2011:13:139-48.

31. Misra S, Pandey AK, Gupta S, Kumar A, Khanna P, shankar J, et al. Estrogen related receptor is required for the testicular development and for the normal sperm axoneme/mitochondrial derivatives in Drosophila males. Scientific Reports. 2017;7. doi:https://doi.org/10.1038/srep40372.

32. Eichner $L$, Giguère $V$. Estrogen related receptors (ERRs): a new dawn in transcriptional control of mitochondrial gene networks. Mitochondrion. 2011;11:544-52.

33. Sladek R, Bader J-A. The orphan nuclear receptor estrogen-related receptor $\checkmark$ is a transcriptional regulator of the human medium-chain acyl coenzyme a dehydrogenase gene. Mol Cell Biol. 1997;17:10.

34. Chen Y, Zhou Z, Min W. Mitochondria, oxidative stress and innate immunity. Front Physiol. 2018;9. https://doi.org/10.3389/fphys.2018.01487.

35. O'Neill LAJ. A broken Krebs cycle in macrophages. Immunity. 2015;42:393-4.

36. Park S, Juliana C, Hong S, Datta P, Hwang I, Fernandes-Alnemri T, et al. The mitochondrial antiviral protein MAVS associates with NLRP3 and regulates its Inflammasome activity. J Immunol. 2013;191:4358-66.

37. Angajala A, Lim S, Phillips JB, Kim J-H, Yates C, You Z, et al. Diverse roles of mitochondria in immune responses: novel insights into Immunometabolism. Front Immunol. 2018:9. https://doi.org/10.3389/fimmu.2018. 01605.

38. Sun W, Valero MC, Seong KM, Steele LD, Huang I-T, Lee C-H, et al. A Glycine insertion in the estrogen-related receptor (ERR) is associated with enhanced expression of three cytochrome P450 genes in transgenic Drosophila melanogaster. PLoS One. 2015;10:e0118779.

39. Östberg T, Jacobsson M, Attersand A, Mata de Urquiza A, Jendeberg L. A Triple Mutant of the Drosophila ERR Confers Ligand-Induced Suppression of Activity ${ }^{\dagger}$. Biochemistry. 2003:42:6427-35.
40. Ferrandon D, Imler J-L, Hetru C, Hoffmann JA. The Drosophila systemic immune response: sensing and signalling during bacterial and fungal infections. Nat Rev Immunol. 2007;7:862-74.

41. Wu L, Silverman N. Fighting infection Fly-style. Fly. 2007;1:106-9.

42. Regan JC, Brandão $A S$, Leitão $A B$, Mantas Dias ÂR, Sucena É, Jacinto $A$, et al. Steroid hormone signaling is essential to regulate innate immune cells and fight bacterial infection in Drosophila. PLoS Pathog. 2013;9:e1003720.

43. Thummel CS. Molecular mechanisms of developmental timing in C. elegans and Drosophila. Dev Cell. 2001;1:453-65.

44. Bi E, Pringle JR. ZDS1 and ZDS2, genes whose products may regulate Cdc42p in Saccharomyces cerevisiae. Mol Cell Biol. 1996;16:5264-75.

45. Nehme NT, Liégeois S, Kele B, Giammarinaro P, Pradel E, Hoffmann JA, et al. A model of bacterial intestinal infections in Drosophila melanogaster. PLoS Pathog. 2007;3:e173.

46. Groves JD, Falson P, le Maire M, Tanner MJ. Functional cell surface expression of the anion transport domain of human red cell band 3 (AE1) in the yeast Saccharomyces cerevisiae. Proc Natl Acad Sci. 1996;93:1224550.

47. McFarland J. The nephelometer: an instrument for estimating the number of bacteria in suspensions used for calculating the opsonic index and for vaccines. JAMA. 1907;XLIX:1176-8.

48. Hartmann S, Nusbaum DJ, Kim K, Alameh S, Ho C-LC, Cruz RL, et al. Role of a small molecule in the modulation of cell death signal transduction pathways. ACS Infectious Diseases. 2018. https://doi.org/10.1021/acsinfecdis. 8b00231.

\section{Publisher's Note}

Springer Nature remains neutral with regard to jurisdictional claims in published maps and institutional affiliations.

Ready to submit your research? Choose BMC and benefit from:

- fast, convenient online submission

- thorough peer review by experienced researchers in your field

- rapid publication on acceptance

- support for research data, including large and complex data types

- gold Open Access which fosters wider collaboration and increased citations

- maximum visibility for your research: over $100 \mathrm{M}$ website views per year

At $\mathrm{BMC}$, research is always in progress.

Learn more biomedcentral.com/submissions 